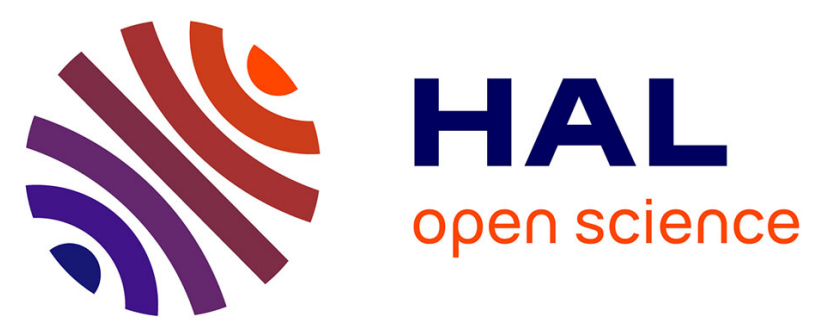

\title{
FiloGen: A Model-Based Generator of Synthetic 3D Time-Lapse Sequences of Single Motile Cells with Growing and Branching Filopodia
}

Dmitry V Sorokin, Igor Peterlik, Vladimír V Ulman, David Svoboda, Tereza Nečasová, Katsiarina Morgaenko, Lívia Eisellová, Lenka Tesařová, Martin Maška

\section{To cite this version:}

Dmitry V Sorokin, Igor Peterlik, Vladimír V Ulman, David Svoboda, Tereza Nečasová, et al.. FiloGen: A Model-Based Generator of Synthetic 3D Time-Lapse Sequences of Single Motile Cells with Growing and Branching Filopodia. IEEE Transactions on Medical Imaging, 2018, pp.1-12. 10.1109/TMI.2018.2845884 . hal-01821338

\section{HAL Id: hal-01821338 \\ https://inria.hal.science/hal-01821338}

Submitted on 19 Jul 2018

HAL is a multi-disciplinary open access archive for the deposit and dissemination of scientific research documents, whether they are published or not. The documents may come from teaching and research institutions in France or abroad, or from public or private research centers.
L'archive ouverte pluridisciplinaire HAL, est destinée au dépôt et à la diffusion de documents scientifiques de niveau recherche, publiés ou non, émanant des établissements d'enseignement et de recherche français ou étrangers, des laboratoires publics ou privés. 


\title{
FiloGen: A Model-Based Generator of Synthetic 3D Time-Lapse Sequences of Single Motile Cells with Growing and Branching Filopodia
}

\author{
Dmitry V. Sorokin, Igor Peterlík, Vladimír Ulman, David Svoboda, Tereza Nečasová, Katsiarina Morgaenko, \\ Lívia Eiselleová, Lenka Tesařová, and Martin Maška Member, IEEE
}

\begin{abstract}
The existence of diverse image datasets accompanied by reference annotations is a crucial prerequisite for an objective benchmarking of bioimage analysis methods. Nevertheless, such a prerequisite is arduous to satisfy for time-lapse, multidimensional fluorescence microscopy image data, manual annotations of which are laborious and often impracticable. In this paper, we present a simulation system capable of generating 3D time-lapse sequences of single motile cells with filopodial protrusions of user-controlled structural and temporal attributes, such as the number, thickness, length, level of branching, and lifetime of filopodia, accompanied by inherently generated reference annotations. The proposed simulation system involves three globally synchronized modules, each being responsible for a separate task: the evolution of filopodia on a molecular level, linear elastic deformation of the entire cell with filopodia, and the synthesis of realistic, time-coherent cell texture. Its flexibility is demonstrated by generating multiple synthetic 3D time-lapse sequences of single lung cancer cells of two different phenotypes, qualitatively and quantitatively resembling their real counterparts acquired using a confocal fluorescence microscope.
\end{abstract}

\section{INTRODUCTION}

$\mathbf{F}$ ILOPODIA are plasma membrane protrusions governed by dynamic bundles of aligned actin filaments. Acting as guidance sensors and promoters of cell locomotion, they play crucial roles in many physiological and pathological processes, such as cell adhesion, chemotaxis, wound healing, embryonic development, or cancer extravasation [1]-[5].

Ever-growing technological advances in optical microscopy and fluorescent reporters have facilitated observations of these processes at unprecedented spatio-temporal resolutions [6], at the expense of increasing amounts of acquired image data to be processed and quantitatively evaluated, calling for robust and fully automatic bioimage analysis solutions [7], [8]. However, objective assessments of bioimage analysis solutions are often arduous, namely due to limited availability of public bioimage

D.V. Sorokin was with the Centre for Biomedical Image Analysis, Faculty of Informatics, Masaryk University, 60200 Brno, Czech Republic. He is currently with the Laboratory of Mathematical Methods of Image Processing, Faculty of Computational Mathematics and Cybernetics, Lomonosov Moscow State University, 119991 Moscow, Russia. He is the corresponding author of this manuscript (e-mail: dsorokin@cs.msu.ru).

I. Peterlík is with the Institute of Computer Science, Masaryk University, 60200 Brno, Czech Republic, and with INRIA, France.

V. Ulman was with the Centre for Biomedical Image Analysis, Faculty of Informatics, Masaryk University, 60200 Brno, Czech Republic. He is currently with the Max Planck Institute of Molecular Cell Biology and Genetics (MPICBG), 01307 Dresden, Germany.

D. Svoboda, T. Nečasová, K. Morgaenko, L. Eiselleová, L. Tesařová, and M. Maška are with the Centre for Biomedical Image Analysis, Faculty of Informatics, Masaryk University, 60200 Brno, Czech Republic. datasets accompanied by reference annotations [9], the preparation of which is known to be subjective, highly error-prone, and extremely laborious, especially when it comes to manually annotating 3D time-lapse image data by human experts [10].

Therefore, recent benchmarks of time-lapse bioimage analysis algorithms [11]-[13] profit from using computer-simulated image datasets, accompanied by inherently generated reference annotations. The simulation platforms adopted for these benchmarks are capable of generating realistic time-lapse sequences of intracellular and intercellular particles [11], and motile cells with nuclear staining [14]. With the primary aim of stimulating the development of fully 3D routines for filopodium segmentation and tracking [15]-[18], a prototype of the simulation system capable of generating 3D time-lapse sequences of single motile cells with filopodial protrusions emerged recently [19]. However, it can only handle a predefined number of curvilinear filopodia with the whole-simulation-period lifetimes, not being capable of mimicking filopodium appearance, disappearance, and branching events, natural for various cell phenotypes [20].

In this paper, we considerably extend the above-mentioned simulation system prototype [19] to be capable of generating realistic 3D time-lapse sequences of single motile cells with filopodial protrusions of user-controlled structural and temporal attributes. The proposed extended simulation system allows one to mimic natural scenarios in which cells asynchronously protrude time-varying numbers of filopodia of different thicknesses, lengths, and levels of branching in response to stimuli from their surrounding environments. It consists of three globally synchronized modules, responsible for stochastic simulations of filopodial evolution [21], simultaneous deformations of the entire cell with filopodia using linear elasticity [22], and the synthesis of realistic, time-coherent cell texture utilizing clouds of simulated fluorescent particles in the spatio-temporal domain [23], the content of which is periodically submitted to a virtual microscope [24]. The proposed simulation system is validated by generating three $3 \mathrm{D}$ time-lapse sequences of lung cancer cells of two different phenotypes. We show that the generated sequences qualitatively and quantitatively resemble their real counterparts acquired using a confocal microscope. Note that the output of the proposed simulation system consists of a 3D synthetic time-lapse sequence and the corresponding labeled masks of the cell body and filopodial branches. Such a reference annotation can directly be used when benchmarking filopodium segmentation and tracking methods, and also when studying to what extent segmentation and tracking errors affect 


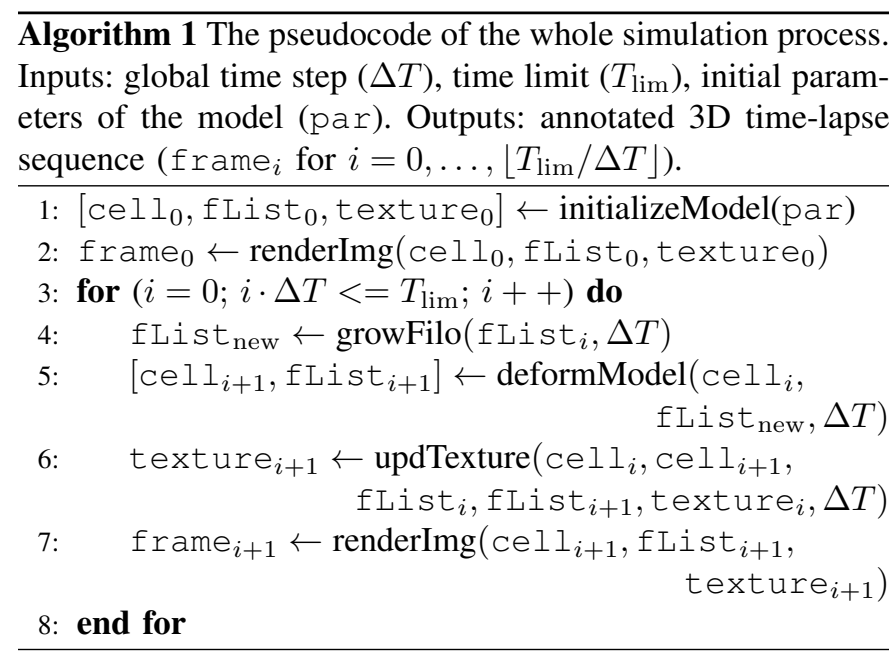

the extraction of quantitative parameters of crawling cells with filopodial protrusions, such as the number, length, and lifetime of filopodia, or their protrusion and retraction rates.

The rest of this paper is organized as follows. The proposed simulation system is described in Section II. Its experimental validation can be found in Section III. Concluding remarks and suggestions for future work are finally given in Section IV.

\section{Methods of the Simulation System}

The simulation of a 3D time-lapse sequence of one motile cell with filopodial protrusions consists of three isolated parts: modeling of filopodium growth and branching (Section II-A), simultaneous elastic deformation of the cell body and individual filopodia (Section II-B), and finally generation of the realistic cell texture (Section II-C). Each part is addressed by an independent software component: geometry module (GM), deformation module (DM), and texture module (TM). The three modules are executed from the main component, a supervisor, that primarily controls the data exchange between the modules, handles their parameters, and synchronizes the time.

The whole simulation process (see Alg. 1) starts by initializing the cell body $\left(\mathrm{cell}_{0}\right)$ and individual filopodia $\left(f \mathrm{List} \mathrm{f}_{0}\right)$, setting their growth parameters, initial lengths $l_{0}$, and the positions where they are attached to the cell body, generating the initial cell texture (texture $e_{0}$ ), and finally rendering the first frame of the time-lapse sequence $\left(\right.$ frame $\left._{0}\right)$. In our data representation, each cell body, cell $l_{i}$, includes only its geometry stored as a tetrahedral mesh, whereas each filopodium in fList ${ }_{i}$ is represented by a tree structure that contains the data related to the filopodium geometry and evolution (see Section II-A4 for more details).

After the initialization, the simulation process continues iteratively. In each iteration, first, new filopodia may appear or some of the existing ones may disappear, depending on predefined probabilities $P_{a}$ and $P_{d}$. Filopodium appearance is implemented by adding a new element to $\mathrm{fList}_{i}$ and running the initialization procedure for it. Filopodium disappearance is implemented by the 5-fold increase of its retrograde flow (see Section II-A for more details), leading to a natural retraction of the filopodium into the cell body. Second, the morphology of individual filopodia is evolved ( $\mathrm{fList} \mathrm{Lew}_{\text {new }}$ ), which allows their

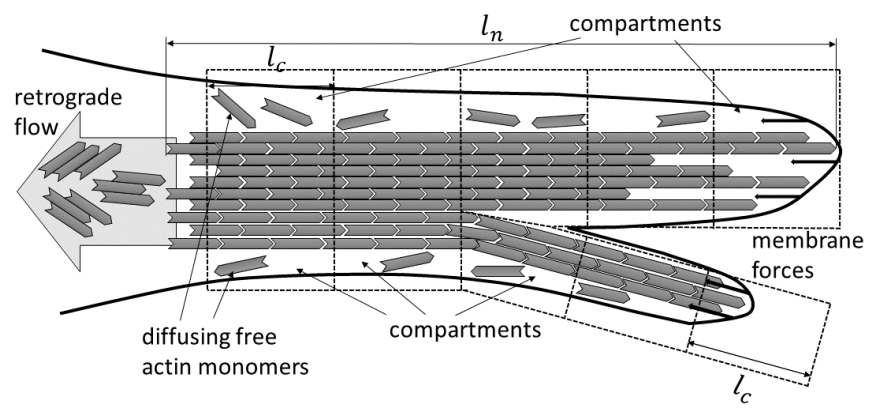

Fig. 1. An illustration of the molecular model of an evolving filopodium, with the compartment size $l_{c}$ and the filament length $l_{n}$. Note that the numbers of filaments and of free actin monomers were purposely decreased compared to the real model values to make the figure content visually simple and intuitive.
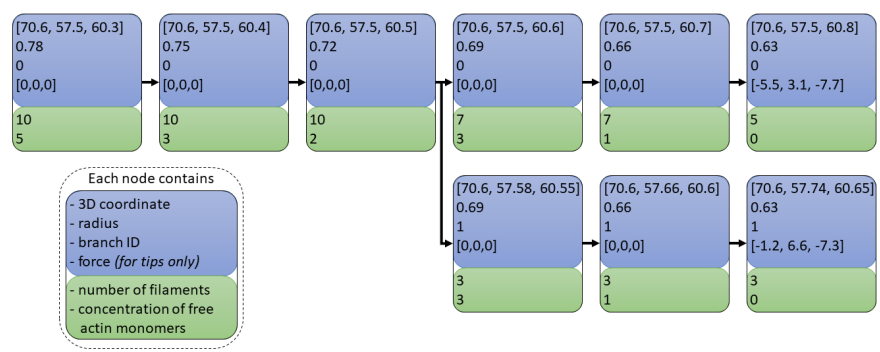

Fig. 2. The fTree structure representing the purely illustrative model of the filopodium in Fig. 1. The upper part of each node contains the information for geometry modeling and deformation, the lower part contains the information for filopodium evolution.

branching or the changes in their lengths and widths. Third, the cell body $\left(\mathrm{cell}_{i}\right)$ and the evolved filopodia are deformed using pressures applied to the cell body and forces applied to the tips of filopodial branches, yielding the deformed cell body $\left(\mathrm{cell}_{i+1}\right)$ and the deformed filopodia $\left(\mathrm{fList}_{i+1}\right)$. In case a filopodial branch has protruded, a new tree node is appended to its tip. In case it has shortened, the corresponding tree node is removed. After having the geometry of the whole cell updated, the cell texture must be recomputed accordingly, which boils down to updating spatial positions of the simulated fluorescent particles. Finally, the current frame of the simulated sequence (frame $e_{i+1}$ ) is created by rendering the model.

The modules work with their own internal time steps, regularly exporting their particular outputs. A user-defined global time interval $\Delta T$, during which each of the three modules is operating, corresponds to the time step of the generated timelapse sequence. The simulation process is terminated after the total accumulated time reaches a pre-defined limit $T_{\text {lim }} \geq \Delta T$.

The labeled masks of the cell body and filopodial branches, establishing the ground truth for the generated 3D time-lapse sequence, are formed by projecting and rasterizing the meshes of the cell body and filopodial branches to the image domain.

\section{A. Modeling of filopodium growth and branching}

In this section, the mathematical models, data structures, and algorithms employed in the geometry module are described.

1) Filopodium evolution on molecular level: A filopodium is a bundle of $n_{f}$ actin filaments enclosed by the cell membrane [2] (see Fig. 1). Its length is defined as the length 
of the longest actin filament $l=\max \left(l_{n}\right)$ where $n$ is the actin filament index. Each actin filament consists of actin monomers connected with a half-monomer-size overlap of $2.7 \mathrm{~nm}$ [21]. The evolution of actin filaments is affected by four processes: diffusion of free actin monomers along the filopodium, polymerization and depolymerization of actin filaments, fluctuation of the membrane under load, slowing down the polymerization rate of actin filaments, and a constantvelocity retrograde flow that continuously pulls actin filaments into the cell body, modeled on a molecular level using the Gillespie algorithm [21].

The filopodium volume is discretized by dividing it into $J$ compartments, with the compartment length of $l_{c}=50 \mathrm{~nm}$. Let $a_{j}, j=1, \ldots, J$, denote the number of actin monomers in the $j$-th compartment, being indexed from the place where the filopodium attaches to the cell body (filopodium base). The diffusion of each free actin monomer is defined as 1D random walk [25] with reflective boundary conditions at the filopodium tips $\left(P_{1 \rightarrow 0}=0\right)$. At the filopodium base, the number of actin monomers remains unchanged at the constant bulk value $\left(a_{0}=\right.$ 5 ) because the number of free actin monomers in the cell body is significantly higher than in the filopodium. Since free actin monomers move between neighboring compartments and stay in the same compartment with equal probability, the reaction time of free actin monomer diffusion is defined as $\Delta t=\frac{l_{c}^{2}}{3 D}$, with $D$ being the diffusion constant [25].

The actin filament polymerization rate is given [21] as

$$
k_{n}=k_{0} \exp \left(-f_{n} \delta /\left(k_{B} T\right)\right)
$$

where $k_{0}$ is the "bare" filament polymerization rate and the exponential term accounts for the probability of opening the gap larger than $\delta$ between the tip of the $n$-th filament and the fluctuating membrane. The term $k_{B} T$ represents the thermal energy and $f_{n}$ is the membrane loading force defined for every filament depending on membrane fluctuations [21]:

$$
f_{n}=\frac{\operatorname{erfc}\left(l-l_{n}, \sigma\right)}{\sum_{i} \operatorname{erfc}\left(l-l_{i}, \sigma\right)} f
$$

where $\sigma$ is the average membrane fluctuation amplitude and $f$ is the total membrane force. The actin filament depolymerization rate does not depend on the membrane loading force and has the same value $k_{d}$ for all filaments.

The motion equation for each filament length $l_{n}$ is given as:

$$
\Delta l_{n}=-\nu_{\text {retr }} \Delta t+\xi \delta-\eta \delta
$$

where $\Delta l_{n}$ is the change of filament length over the reaction time $\Delta t, \nu_{\text {retr }}$ is the retrograde flow velocity, and $\xi$ and $\eta$ are the binary indicators of polymerization and depolymerization events, respectively.

Every iteration of the Gillespie simulation involves three steps [26]. First, two random numbers, $r_{1}$ and $r_{2}$, uniformly distributed in the $[0,1]$ interval are generated. Next, assuming $k_{d} \leq k_{n}$ without loss of generality, the type of event to occur in a particular iteration is established as depolymerization $(\xi=0 ; \eta=1)$ if $0 \leq r_{1}<\frac{k_{d}}{k_{d}+k_{n}}$, polymerization $(\xi=1$; $\eta=0)$ if $\frac{k_{d}}{k_{d}+k_{n}} \leq r_{1}<\frac{k_{n}}{k_{d}+k_{n}}$, or free actin monomer diffusion $(\xi=\eta=0)$ if $r_{1} \geq \frac{k_{n}}{k_{d}+k_{n}}$. Finally, depending on the event type, the number of free actin monomers in each compartment is updated, and the reaction time $\Delta t$ is calculated as either $\Delta t=l_{c}^{2} /(3 D)$ in case of diffusion or $\Delta t=\left(a_{n}^{\mathrm{tip}}\left(k_{d}+k_{n}\right)\right)^{-1} \ln \left(1 / r_{2}\right)$ in case of polymerization or depolymerization, with $a_{n}^{\text {tip }}$ being the number of free actin monomers in the filopodium compartment that corresponds to the tip of the $n$-th filament, not necessarily being the first one. The Gillespie simulations are performed individually for each actin filament over the global time step $\Delta T$.

2) Filopodium branching: The filaments in the bundle are tied together by special binding proteins. In case the proteins are not up to tie the complete bundle together, the filopodium branches. Without loss of generality, we assume that it happens at the interface of two successive compartments (see Fig. 1). The new branch volume is again discretized by dividing it into the compartments of the length $l_{c}$, each having its own number $a_{j}$ of free actin monomers. The values of $a_{j}$ are changing over the Gillespie simulations for the filaments that are located in the corresponding compartments of the new branch.

The branching events depend on the pre-defined probability $P_{b}$. In our simulation system, every new branch contains a random portion of the filaments from the mother branch, and the rest of the filaments remain in the mother branch. The angle between the new branch and the mother branch is given by a random variable with the uniform distribution $\mathcal{U}\left(50^{\circ}, 70^{\circ}\right)$.

3) Filopodium shape: In order to model the 3D shape of a filopodium, every filopodium tree node has a radius assigned, which linearly decreases from the base point to the tips. The radius at the filopodium base is represented by a random variable with uniform distribution $\mathcal{U}\left(r_{m}-r_{\varepsilon}, r_{m}+r_{\varepsilon}\right)$, where $r_{m}$ and $r_{\varepsilon}$ are pre-defined parameters. The radii at the filopodial tips correspond to a third of that at the filopodial base.

4) Data structures: Every filopodium is represented by a tree structure composed of $J+1$ nodes, one for each compartment and the root that corresponds to the filopodium base. Each node contains the information related to the filopodium geometry (3D coordinate of the filopodium base for the root node, of the filopodial tip for a leaf node, and of the end of the corresponding compartment for the other nodes; radius at that spatial coordinate; branch ID; and force applied to the node) and to the filopodium evolution (the number of filaments and the concentration of free actin monomers), as shown in Fig. 2. Furthermore, for every filopodium, a list of $n_{f}$ elements, one for each actin filament of the filopodium, is maintained. Each element contains the information about the filament length and the index of the compartment that contains the filament tip.

5) Overall simulation of the filopodial evolution: For each filopodium, given by the corresponding tree structure (fTree) and list of filaments ( $\mathrm{flm}$ ), the simulation process (see Alg. 2) runs with a non-uniform, a priori unknown time step due to a possible occurrence of different events (i.e., diffusion, polymerization, or depolymerization) in the Gillespie algorithm. Each iteration starts by checking the occurrence of filopodium branching, being performed at most once per the global time step $\Delta T$. Next, each filament $\left(f_{n}\right)$ of the filopodium is evolved using the Gillespie algorithm and its updated version $\left(f_{n}^{\text {new }}\right)$ is propagated back to the corresponding data structures (fTree and $f l m)$. The iterative process finishes after the internal time 


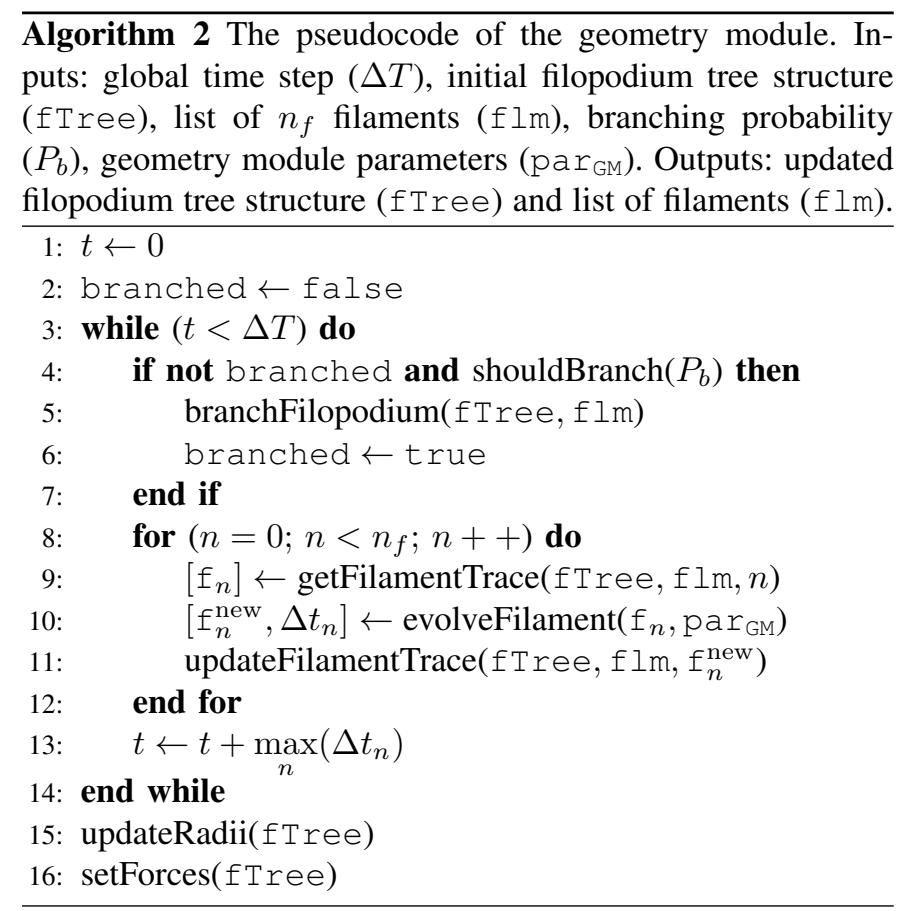

( $t)$ reaches the global time step $\Delta T$. Finally, the information about the filopodium radius in each compartment and about the forces applied to the filopodial tips is updated accordingly, thus providing the deformation module with the actual information needed to properly deform the evolved filopodium.

\section{B. Deformation of cell body and filopodia}

The motion and deformation of the cell is modeled by the deformation module, which is started just after geometry module every global time step. To mimic realistic deformations, the cell body and the filopodia are modeled as elastic objects coupled together via mechanical constraints. Each filopodium and the cell body has its proper dynamics based on the elastic formulation discretized by the finite element method (FEM). The mechanical coupling between a filopodium base and the cell body propagates the excitations in both directions: forces applied to filopodia influence the motion of the cell body and random pressures applied to the cell body surface result in the motion of filopodia. We first characterize the dynamic formulation of filopodial and cell body motions and then, we describe the mechanical coupling.

1) Mechanical model of filopodia: Each filopodium is modeled as a deformable tree of piecewise linear segments of nonzero radii, represented by linear beam elements based on the Timoshenko formulation [27]. While being computationally efficient, the beam elements are typically used to model threadlike structures which undergo bending and display important force responses when subjected to longitudinal deformations.

Let a filopodium $\varphi$, represented by a fTree structure, be given as a set of nodes $\left\{\mathbf{q}_{0}, \ldots \mathbf{q}_{n}\right\}$, each being an oriented point in 3D space with three positional and three rotational degrees of freedom (DoF). Its dynamics is governed by the Newton's law of motion relating the body acceleration and forces:

$$
\mathbf{M}_{\varphi} \ddot{\mathbf{q}}=\mathbf{f}_{\varphi}-\mathbf{g}_{\varphi}(\mathbf{q}, \dot{\mathbf{q}})+\mathbf{H}_{\varphi}^{\top} \boldsymbol{\lambda}
$$

where $\mathbf{M}_{\varphi}$ is an inertia matrix, $\mathbf{q}$ is a vector of filopodium node coordinates, $\mathbf{f}_{\varphi}$ is a vector of external forces, and $\mathbf{g}_{\varphi}$ is a vector of internal elastic forces. The term $\mathbf{H}_{\varphi}^{\top} \boldsymbol{\lambda}$ represents the coupling between the filopodium and cell body, explained in more detail in Section II-B3.

The internal forces are represented as a general non-linear function that is linearized in each internal time step of the deformation module:

$$
\mathbf{g}_{\varphi}(\mathbf{q}+d \mathbf{q}, \dot{\mathbf{q}}+d \dot{\mathbf{q}}) \approx \mathbf{g}_{\varphi}(\mathbf{q}, \dot{\mathbf{q}})+\frac{\partial \mathbf{g}_{\varphi}}{\partial \mathbf{q}} d \mathbf{q}+\frac{\partial \mathbf{g}_{\varphi}}{\partial \dot{\mathbf{q}}} d \dot{\mathbf{q}}
$$

The derivatives $\mathbf{K}_{\varphi}=\frac{\partial \mathbf{g}_{\varphi}}{\partial \mathbf{q}}$ and $\mathbf{B}_{\varphi}=\frac{\partial \mathbf{g}_{\varphi}}{\partial \dot{\mathbf{q}}}$ are known as stiffness and damping matrices, respectively. In the actual scenario, the inertia matrix $\mathbf{M}_{\varphi}$ is constant and diagonal. Since we employ the co-rotational formulation of each beam element [27], [28], the stiffness matrix $\mathbf{K}_{\varphi}$ is updated in each internal time step because the rotational components depend on the actual deformation. The stiffness matrix $\mathbf{K}_{\varphi}$ determines the physical behavior of the filopodium and is parametrized by Young's modulus $E_{\varphi}$ and Poisson ratio $\nu_{\varphi}$. The damping matrix $\mathbf{B}_{\varphi}$ is approximated using Rayleigh stiffness $r_{K}$ and Rayleigh mass $r_{M}$, as $\mathbf{B}_{\varphi}=r_{K} \mathbf{K}_{\varphi}+r_{M} \mathbf{M}_{\varphi}$. The vector of external forces $\mathbf{f}_{\varphi}$ represents the excitation forces applied to the filopodial tips. For every filopodial branch, the force is applied to its tip in random direction orthogonal to its last segment, and the force magnitude is given as the simulation parameter $F_{\text {tip }}$.

The integration of (1) is carried out using the implicit Euler scheme [29] computed with an internal time step $h$. Denoting $t$ the previous DM time step, the linear system in the actual DM time step $t+h$ is given [30] as:

$$
\underbrace{\left(\mathbf{M}_{\varphi}-h \mathbf{B}_{\varphi}-h^{2} \mathbf{K}_{\varphi}\right)}_{\mathbf{A}_{\varphi}} d \dot{\mathbf{q}}=\underbrace{h \mathbf{g}_{\varphi}-h^{2} \mathbf{K}_{\varphi} \dot{\mathbf{q}}^{t}+\mathbf{f}_{\varphi}}_{\mathbf{b}_{\varphi}}+h \mathbf{H}_{\varphi}^{\top} \boldsymbol{\lambda}
$$

where $\mathbf{M}_{\varphi}$ is constant, and $\mathbf{B}_{\varphi}, \mathbf{K}_{\varphi}$, and $\mathbf{g}_{\varphi}$ are computed using the position $\mathbf{q}^{t}$ obtained in the previous internal time step. The linear system is solved by the square-root-free Cholesky decomposition in the form of $\mathbf{A}_{\varphi}=\mathbf{L D L}^{\top}$, with $\mathbf{L}$ being a unit lower triangular matrix and $\mathbf{D}$ being a diagonal matrix.

2) Mechanical model of the cell body: We use the same approach based on the implicit Euler method to model the cell body, however, unlike in the case of filopodia, the cell body is modeled using 3D co-rotational elasticity discretized by linear tetrahedra. Therefore, the cell body domain is given by a set of nodes $\left\{\mathbf{p}_{0}, \ldots, \mathbf{p}_{m}\right\}$ where each $\mathbf{p}_{i}$ is given by three positional degrees of freedom. The discretized Newton's equation of cell body motion is

$$
\mathbf{M}_{c} \ddot{\mathbf{p}}=\mathbf{f}_{c}-\mathbf{g}_{c}(\mathbf{p}, \dot{\mathbf{p}})+\mathbf{H}_{c}^{\top} \boldsymbol{\lambda}
$$

where $\mathbf{M}_{c}$ is the constant inertia matrix, $\mathbf{p}$ is a vector of cell body node coordinates, and $\mathbf{f}_{c}$ and $\mathbf{g}_{c}$ are the external and internal forces of the cell body, respectively. The term $\mathbf{H}_{c}^{\top} \boldsymbol{\lambda}$ represents the coupling between the cell body and the filopodia, explained in more detail in Section II-B3. The same linearization procedure is applied as in the case of filopodium: given the constant matrix $\mathbf{M}_{c}$ and $\mathbf{K}_{c}, \mathbf{B}_{c}, \mathbf{f}_{c}$ and $\mathbf{g}_{c}$ computed 
using the positions $\mathbf{p}^{t}$ obtained in the previous DM time step, the dynamic system in the DM actual step $t+h$ is given as

$$
\underbrace{\left(\mathbf{M}_{c}-h \mathbf{B}_{c}-h^{2} \mathbf{K}_{c}\right)}_{\mathbf{A}_{c}} d \dot{\mathbf{p}}=\underbrace{h \mathbf{g}_{c}-h^{2} \mathbf{K}_{c} \dot{\mathbf{p}}^{t}+\mathbf{f}_{c}}_{\mathbf{b}_{c}}+h \mathbf{H}_{c}^{\top} \boldsymbol{\lambda} .
$$

The global stiffness matrix $\mathbf{K}_{c}$ is assembled from local matrices $\mathbf{K}_{c_{\tau}}$; each $\mathbf{K}_{c_{\tau}}$ computed for a tetrahedron $\tau$ is a $4 \times 4$ block matrix where each block is of size $3 \times 3$ and is determined by Young's modulus $E_{c}$ and Poisson ratio $\nu_{c}$ of the cell. Please see [31] for further details about the co-rotated formulation of linear elasticity and the definition of matrix $\mathbf{K}_{c}$. The external forces $\mathbf{f}_{c}$ applied to the cell body result from the random pressures acting on the cell surface [23]. The magnitude of the random pressures is set as the simulation parameter $P_{c b}$.

3) Coupled dynamic simulation of cell with filopodia: Without loss of generality, we describe a system composed of a cell body and one filopodium $\varphi$ and it is supposed that initially the $6 \mathrm{DoF}$ base $\mathbf{q}_{0}^{0}$ of the filopodium is located inside a tetrahedron $\tau_{\varphi}$. During the simulation, the initial relative position and orientation of $\mathbf{q}_{0}^{0}$ with respect to $\tau_{\varphi}$ are maintained: therefore, we introduce mapped base $\mathbf{r}_{\varphi}$ : initially, $\mathbf{r}_{\varphi}=\mathbf{q}_{0}^{0}$ and during the simulation, its position is updated together according to the actual position of the tetrahedron $\tau_{\varphi}$.

The relative position and the orientation of $\mathbf{r}_{\varphi}$ are given by the barycentric coordinates $\beta_{\varphi}$ of $\mathbf{r}_{\varphi}$ with respect to $\tau_{\varphi}$ and the rotation matrix $\boldsymbol{\Omega}_{\varphi}$, which are initially precomputed and remain constant during the simulation [32].

In the actual DM time step $t+h$, the coupled dynamics of the cell with filopodium is computed as follows:

- Free motion: the vector of constraint forces is set to zero $(\boldsymbol{\lambda}=0)$, and (3) and (5) are solved for $d \dot{\mathbf{q}}$ and $d \dot{\mathbf{p}}$, respectively. These are then integrated to obtain positions of both the filopodium and the cell body according to unconstrained dynamics: $\mathbf{q}^{\text {free }}=\mathbf{q}^{t}+h\left(\dot{\mathbf{q}}^{t}+d \dot{\mathbf{q}}\right)$, $\mathbf{p}^{\text {free }}=\mathbf{p}^{t}+h\left(\dot{\mathbf{p}}^{t}+d \dot{\mathbf{p}}\right)$. Since the tetrahedron $\tau_{\varphi}$ has moved, it is necessary to update the mapped filopodium base $\mathbf{r}_{\varphi}$ using the precomputed barycentric coordinates $\beta_{\varphi}$ and its orientation using the rotation matrix $\boldsymbol{\Omega}_{\varphi}$.

- Computation of coupling forces: Due to the independent dynamics computed by the free motion, the actual filopodium base $\mathbf{q}_{0}$ and mapped filopodium $\operatorname{root} \mathbf{r}_{\varphi}$ do not necessarily coincide. However, to maintain the coupling between the two objects, it is necessary to impose $\mathbf{q}_{0}=\mathbf{r}_{\varphi}$, i.e. $\delta=\left|\mathbf{r}_{\varphi}-\mathbf{q}_{0}\right|=0$. This is done by computing the constraint force $\boldsymbol{\lambda}$ that must be applied to both objects in order to achieve the equality $\delta=0$. As shown in [30]

$$
\delta=\delta^{\text {free }}+h\left[\mathbf{H}_{\varphi} \mathbf{A}_{\varphi}^{-1} \mathbf{H}_{\varphi}^{\top}+\mathbf{H}_{c} \mathbf{A}_{c}^{-1} \mathbf{H}_{c}^{\top}\right] \boldsymbol{\lambda}
$$

where $\delta^{\text {free }}$ is a constraint violation after the free motion, and $\mathbf{A}_{\varphi}^{-1}$ and $\mathbf{A}_{c}^{-1}$ are the inverted system matrices computed during the free motion in (3) and (5). The matrices $\mathbf{H}_{\varphi}$ and $\mathbf{H}_{c}$ map the force vector $\boldsymbol{\lambda}$ to the degrees of freedom of respective objects. In the case of filopodium, $\mathbf{H}_{\varphi}$ is a $6 \times 6 N$ matrix $(N$ is the number of nodes in the filopodium), which is zero everywhere except for the leftmost block, corresponding to the first node, which is given as the $6 \times 6$ identity matrix. The $6 \times 3 M$ matrix $(M$ is the number of nodes in the cell body) $\mathbf{H}_{c}$ maps the $6 \mathrm{DoF}$ forces $\boldsymbol{\lambda}$ to four linear forces applied to the nodes of the tetrahedron $\tau_{\varphi}$. Therefore, $\mathbf{H}_{c}$ contains four non-zero blocks of size $6 \times 3$ : one for each vertex $I$ of the tetrahedron $\tau_{\varphi}$. Each block is given as

$$
\mathbf{H}_{c I}^{\top}=\left(\begin{array}{cccccc}
\beta_{I} & 0 & 0 & 0 & -\Delta_{I}^{z} & \Delta_{I}^{y} \\
0 & \beta_{I} & 0 & \Delta_{I}^{z} & 0 & -\Delta_{I}^{x} \\
0 & 0 & \beta_{I} & -\Delta_{I}^{y} & \Delta_{I}^{x} & 0
\end{array}\right)
$$

where $\beta_{I}$ are the barycentric coordinates of $\mathbf{r}_{\varphi}$ and $\Delta_{I}=$ $\mathbf{p}_{I}-\mathbf{r}_{\varphi}$ is the line given by the updated mapped position of the filopodium base and the vertex $I$ of the tetrahedron $\tau_{\varphi}$. The system (6) is solved using the iterative GaussSeidel method.

- Corrective motion: The corrected positions of both the cell body and filopodium are obtained by applying the coupling forces $\boldsymbol{\lambda}$ to the configurations obtained by the free motion. This is done by computing

$$
\begin{aligned}
\mathbf{q}^{t+h} & =\mathbf{q}^{\text {free }}+\mathbf{A}_{\varphi}^{-1} \mathbf{H}_{\varphi} \boldsymbol{\lambda} \\
\mathbf{p}^{t+h} & =\mathbf{p}^{\text {free }}+\mathbf{A}_{c}^{-1} \mathbf{H}_{c} \boldsymbol{\lambda} .
\end{aligned}
$$

4) Cell body motion: At the beginning of the execution of the deformation module on every global time step, the cell centroid coordinates are moved to a new location and rotated by a random angle in $3 \mathrm{D}$ to mimic the random motion of the cell. The cell motion is incorporated to the model by applying the force to the center of the cell body (for translation), and on several nodes close to the center of the cell body (for rotation). Both translational and rotational forces are included in the external forces $\mathbf{f}_{c}$ in (5) thus the other parts of the cell are influenced taking into account the elasticity of the cell which results in motion that resembles amoeboid-like motility. These forces are defined using a trajectory generated beforehand which is a simulation parameter. The cell rotation is defined by the random rotation axis and the random rotation angle $\gamma$, which is also a simulation parameter.

\section{Generation of cell texture}

The cell texture is treated with three main objectives in mind: high realism of the texture, ability to adapt to cell shape changes, and visual coherence between consecutive frames of the generated time-lapse sequence. Two different paradigms are employed. For the cell body, which is not changing its volume rapidly, a texture visual representation is properly initiated for the first time point, and is updated according to tracked changes in cell geometries in consecutive time points. For every filopodial branch, which besides bending is also growing or retracting, a new texture visual representation is created for every time point.

The visual representation of the simulated cell is implemented using a set of numerous texture particles where each particle represents a clump of fluorescent molecules [14], [23], [33]. This is a consequence of the fluorescence microscopy 



Fig. 3. Illustration of the data processed during the pipeline of the TM module: Surface of an input mesh, rasterized as a labeled mask, obtained phantom image and final output image. All images are $z$-axis maximum projections and are enhanced for visual purposes. The voxel size is $0.126 \times 0.126 \mu$.

principle that only cell parts stained with a particular fluorescent dye (GFP, Hoechst, etc.) are visible in acquired images. In this study, we mimic transient cell transfection with the green fluorescent protein conjugated to actin. Such a labeling, required for a good definition of the filopodial structures, produces a heterogeneous fluorescent signal within the cell body volume, being concentrated in the cortical cytoskeleton of the cell and leaving the inner parts of the cell practically unstained.

Every texture particle is represented by a floating-point micrometer-scaled three-dimensional coordinate vector, and initiated with a fixed amount of fluorescence intensity it carries. Projecting all particles together into a 3D lattice (of generally anisotropic resolution) and accumulating their fluorescence gives rise to a digital phantom image $I: \mathbb{Z}^{3} \rightarrow \mathbb{N}_{0}$ (see Fig. 3).

To address different geometry developments and labeling outcomes for cell body and for filopodial branches, we treat the ensemble of texture particles in each case separately.

1) Cell body: To initiate the set of texture particles, a cell body mask is created, filled with a Perlin noise [34], and particles are sampled from this. To create the mask, the volumetric tetrahedral mesh from DM is superimposed over the 3D lattice to give rise to a cell body mask image $M_{b}: \mathbb{Z}^{3} \rightarrow\{0,1\}$ in which 1 is set to voxels that intersect with the volume of the mesh, and 0 otherwise.

Perlin noise [34] yields a coherent procedural texture image $\rho: \mathbb{Z}^{3} \rightarrow \mathbb{R}$ controlled by four parameters influencing its coarseness $C$, smoothness $S$, flickering $F l$, and fineness $F i$. The coarseness $C$ is a number controlling the magnification of the blobs in the texture and we introduce it to make our model scalable. The remaining three parameters are introduced in [34]. The Perlin texture is composed of periodic functions, i.e., the domain of texture is unlimited. In order to always get a unique texture image we have to apply a random coordinate shift $S[$.$] on the generated texture \rho$. This type of texture images is commonly accepted as a good approximation of randomized cellular structures [35].

A mosaic of Perlin noises is used to model the initial texture of a cell body. Inner part of the cell body has very fine and dark structure. This part is generated using a fine-grained Perlin noise $\rho_{c i}$ (parameters $C_{c i}, S_{c i}, F l_{c i}$, and $F i_{c i}$ ):

$$
\tilde{I}_{c i}=S\left[\rho_{c i}\right] \text {. }
$$

Compared to the inner structure, the boundary of the cell body (which corresponds to the cortical part) is much brighter and coarser. Here, we employ a coarse-grained Perlin noise $\rho_{c b}$ (parameters $C_{c b}, S_{c b}, F l_{c b}$, and $F i_{c b}$ ):

$$
\tilde{I}_{c b}=S\left[\rho_{c b}\right] .
$$

To create a texture image $\tilde{I}$ to initialize texture particles with, a linear blending between $\tilde{I}_{c b}$ and $\tilde{I}_{c i}$ is performed and the product is masked with $M_{b}$. Let $w$ be the width of the cell body's perimeter volume that represents the cell boundary with coarser texture, let $s$ be the width of a transition strip between the two textures, and EDM be the Euclidean distance map of the foreground voxels in $M_{b}$. The texture image is then defined as:

$$
\tilde{I}(\mathbf{x})=\left(\alpha(\mathbf{x}) \cdot \tilde{I}_{c i}(\mathbf{x})+(1-\alpha(\mathbf{x})) \cdot \tilde{I}_{c b}(\mathbf{x})\right) \cdot M_{b}(\mathbf{x}),
$$

where $\alpha(\mathbf{x})=\min (s, \max (0, \operatorname{EDM}(\mathbf{x})-w)) / s$ (in this work we used $w=0.1 \mu \mathrm{m}$ and $s=0.05 \mu \mathrm{m})$. To obtain an initial distribution of particles in the cell body, the particles are introduced for every texture voxel of the image $\tilde{I}$ in a number that is proportional to that voxel intensity. The set of all cell body particles' coordinates is marked as $\left\{\tilde{\mathbf{p}}_{i}\right\}$ and its size is $\left|\left\{\tilde{\mathbf{p}}_{i}\right\}\right|=\sum_{\mathbf{x}}\lceil\tilde{I}(\mathbf{x}) / Q\rceil$, with $Q$ being a pre-defined quantization factor. The number $\left|\left\{\tilde{\mathbf{p}}_{i}\right\}\right|$ is constant throughout the entire simulation.

The geometry of the cell body is represented with volumetric tetrahedral mesh. The GM provides initial body mesh which is further deformed by DM every global time step based on the elastic behavior of the cell. This way, the DM inherently generates the displacements in all mesh vertices. By interpolating the displacements between the mesh vertices one can form a vector field that represents geometry change from the previous to the current time point. The coordinates $\tilde{\mathbf{p}}_{i}$ of the texture particles, fitting originally to the geometry for the previous time point, are updated according to this vector field. We have previously shown that applying this principle to the texture representation satisfies the three objectives [23] above. Since the deformation field generated by the DM is smooth due to the chosen elasticity model, the update of texture cannot introduce abrupt changes in voxel intensities. However, the condensation or spread of the texture, leading to a local increase or decrease in voxel intensities, can occur 
in case the corresponding part of the cell volume changes, for example nearby protruding cell membrane.

2) Filopodial branch: Geometry of each filopodial branch $\varphi$ is fed into the TM in the form of a Timoshenko beams, that is, as a list of points $\mathbf{q}_{i}$ that sample the beams, with radius $r_{i}$ associated to every point. This geometry can also be understood as a piece-wise conical object that poses a base-totip central axis and whose width decreases from the base to the tip. Indeed, a surface mesh representing the filopodial branch is built and rasterized (see Fig. 3) into a mask image $M_{\varphi}$ : $\mathbb{Z}^{3} \rightarrow\{0,1\}$ in which 1 is set to voxels that are inside this mesh, and 0 otherwise. Considering any conical segment of the filopodium, e.g., between points $\mathbf{q}_{k}$ and $\mathbf{q}_{k+1}$, two circles are constructed, sampled with 10 points and surface triangles are created between two consecutive points on one circle and one point on the other circle. A circle centered at point $\mathbf{q}_{k}$ is of radius $r_{k}$ and coincides with a plane with a normal vector $\mathbf{q}_{k+1}-\mathbf{q}_{k-1}$. The normal vectors used for circles associated with the first and last points of the filopodium branch are colinear with the first and last segments, respectively.

The coordinates $\tilde{\mathbf{q}}_{i}$ of texture particles representing a filopodium $\varphi$ are introduced in the similar fashion. In real image data, the filopodia are visually recognized as bright compartments without an apparent internal structure. To achieve the same visual experience, the volume of a filopodium is populated with a larger number of uniformly distributed particles, making the resulting texture insensitive to particular precise coordinates of the individual particles. Any beam is, therefore, regularly sampled in $\left\lceil\left|\mathbf{q}_{k}-\mathbf{q}_{k+1}\right| / M_{\text {axial }}\right\rceil$ steps (see Table I for the parameter $M_{\text {axial }}$ value), and a disc centered at every sampling point is assumed. The disc is further assumed perpendicular to the axis at the point, and with radius $r$ linearly interpolated between $r_{k}$ and $r_{k+1}$. Finally, the disc is populated with $M_{\text {radial }} \cdot r^{2}$ particles, where $M_{\text {radial }}$ is a constant influencing the density of particles in this disc (see Table I). Iterating over all sampling points and over all beams, the set of all filopodial branch particles' coordinates $\left\{\tilde{\mathbf{q}}_{i}\right\}$ is created. Due to the changes in the geometry and volume of the filopodium, the number of created particles usually changes between time steps, but their density is maintained. This allows the texture particles to be always created from scratch to the current filopodium geometry in every time point without compromising visual coherency of the filopodium texture in successive time points.

For any time point, it now remains to construct output images. The labeled mask is obtained as a weighted sum of all the mutually disjoint masks $M_{b}$ and $M_{\varphi}$, where the weights are the expected numerical labels of individual components. The phantom image $I(\mathbf{x})$ is constructed using union $\left\{\tilde{\mathbf{p}}_{i}\right\} \cup\left\{\tilde{\mathbf{q}}_{i}\right\}$ of the sets with texture particles' coordinates that existed at the respective time point:

$$
I(\mathbf{x})=\sum_{\zeta \in\left\{\tilde{\mathbf{p}}_{i}\right\} \cup\left\{\tilde{\mathbf{q}}_{i}\right\}} \chi(\zeta, \mathbf{x})
$$

where $\chi(\zeta, \mathbf{x})=Q$ if particle $\zeta$ falls into voxel $\mathbf{x}$, otherwise $\chi(\zeta, \mathbf{x})=0$.

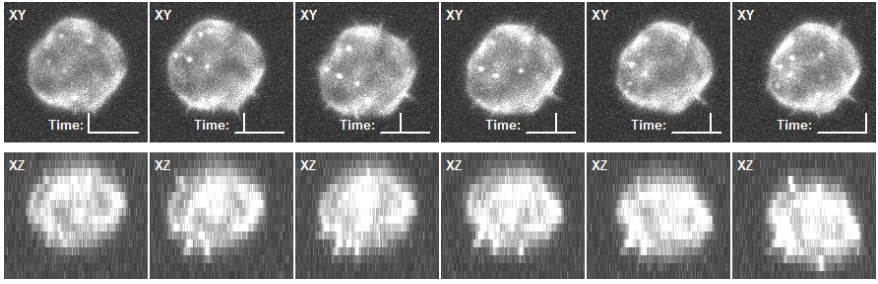

Fig. 5. Two maximum intensity projections of a synthetic 3D time-lapse sequence of an overexpressing cell. The voxel size is $0.126 \times 0.126 \times 1.0 \mu \mathrm{m}$.

\section{Simulation of optical system and acquisition device}

To obtain the final image that is visually similar to a real microscopy one, the phantom image $I$ is submitted to a virtual microscope [36]. In particular, the phantom image is first blurred by an experimental point spread function, measured on a real optical system, and second affected by an uneven illumination phenomenon [37]. Next, it is submitted to a virtual CCD camera. The uncertainty of the photo detection in the camera is modeled with photon shot noise that is subject to Poisson distribution. Finally, the image is affected by amplifier read out, and dark current noise, all of them according to the camera documentation (see Fig. 3). As the final step, the anisotropy of the final synthetic image is obtained by subsampling the synthetic image along $z$-axis.

\section{RESULTS}

To demonstrate the capability and flexibility of the proposed simulation system, we imitated actin-stained A549 lung cancer cells stably transfected with two genetically different variants of the CRMP-2 protein: overexpressing and phospho-defective, which are the representatives of mesenchymal migration typical for cancer extravasation [5]. Depending on its phosphorylation status, CRMP-2 is a cytosolic phosphoprotein that partly contributes to the microtubule dynamics by either promoting or impairing the ability of adding tubulin heterodimers to the microtubule plus ends. Therefore, it indirectly affects filopodial formation via microtubule scaffolding that bidirectionally interacts with actin filaments, both actively regulating the cell cytoskeleton shape [1].

As a reference, we considered real image data with the voxel size of $0.126 \times 0.126 \times 1.0 \mu \mathrm{m}$, being acquired every two minutes over one hour using a spinning disk confocal microscope equipped with a water Plan-Apochromatic $63 \times / 1.20$ objective lens [20]. Whereas the overexpressing cells had variable cell body texture and protruded very short, single-branch filopodia of low motility that frequently appeared and disappeared, the phospho-defective cells had less heterogeneous cell body texture and protruded long, branching filopodia of high motility with whole-experiment lifetimes. As a counterpart to three real 3D time-lapse sequences of each of the two cell phenotypes, we generated three synthetic 3D time-lapse sequences of either cell phenotype using the proposed simulation system. In total, we analyzed 180 real and 180 synthetic images to qualitatively and quantitatively evaluate the similarity between the real and computer-generated image data. 

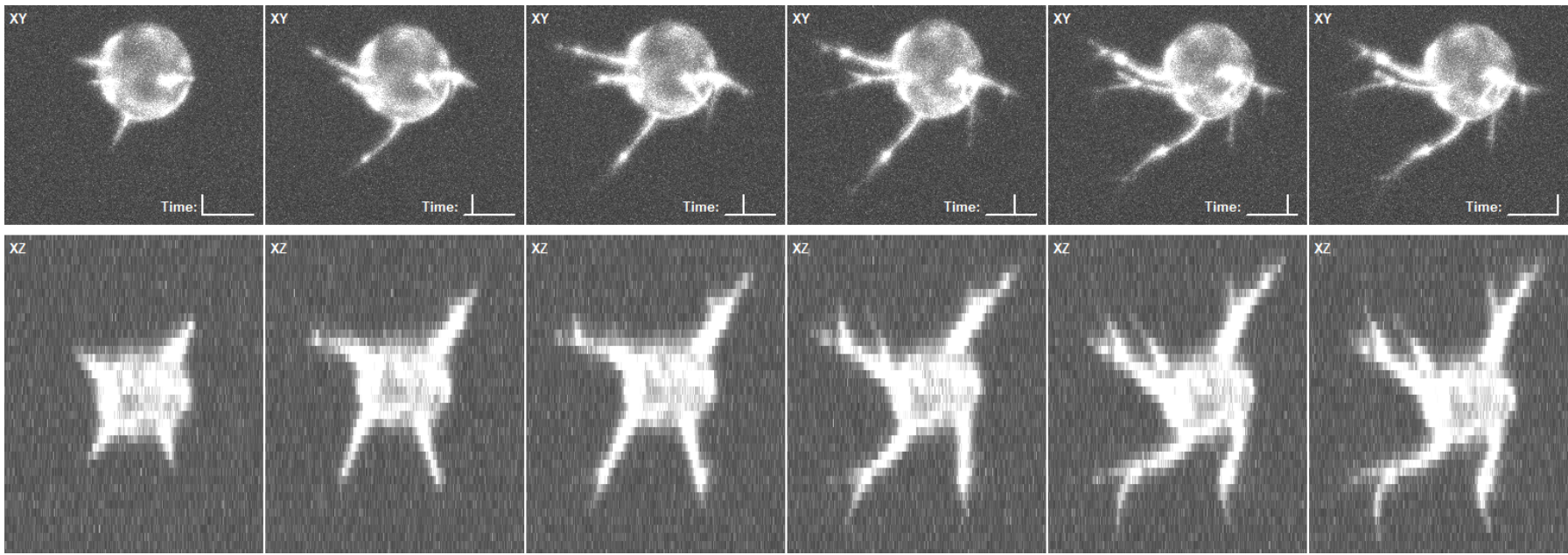

Fig. 4. Two maximum intensity projections of a synthetic 3D time-lapse sequence of a phospho-defective cell. The voxel size is $0.126 \times 0.126 \times 1.0 \mu \mathrm{m}$.

TABLE I

PARAMETER VALUES USED FOR GENERATING SYNTHETIC 3D TIME-LAPSE SEQUENCES OF OVEREXPRESSING AND PHOSPHO-DEFECTIVE CELLS.

\begin{tabular}{|c|c|c|c|c|}
\hline Group & Name & Overexpres. & Phospho-def. & Unit \\
\hline \multirow[t]{3}{*}{ Global } & $\Delta T$ & 20 & 20 & $\mathrm{~s}$ \\
\hline & $T_{\lim }$ & 580 & 580 & $\mathrm{~s}$ \\
\hline & $N_{\varphi}$ & 12 & 5 & \\
\hline \multirow{5}{*}{ Shape } & $P_{b}$ & 0 & 0.5 & \\
\hline & $P_{a}$ & 0.5 & 0 & \\
\hline & $P_{d}$ & 0.5 & 0 & \\
\hline & $r_{m}$ & 0.25 & 1 & $\mu \mathrm{m}$ \\
\hline & $r_{\varepsilon}$ & 0.05 & 0.3 & $\mu \mathrm{m}$ \\
\hline \multirow[t]{9}{*}{ Gillespie } & $D$ & 5 & 5 & $\mu \mathrm{m}^{2} \mathrm{~s}^{-1}$ \\
\hline & $n_{f}$ & 16 & 16 & \\
\hline & $l_{0}$ & 0.081 & $\mathcal{U}(3,4)$ & $\mu \mathrm{m}$ \\
\hline & $k_{d}$ & 1.4 & 1 & $\mathrm{~s}^{-1}$ \\
\hline & $k_{0}$ & 21.8 & 52.4 & \\
\hline & $k_{B} T$ & 4.1 & 4.1 & $\mathrm{pN} \cdot \mathrm{nm}$ \\
\hline & $\sigma$ & 10 & 10 & \\
\hline & $f$ & 10 & $\mathcal{U}(1.5,2.5)$ & $\mathrm{pN}$ \\
\hline & $\nu_{\text {retr }}$ & $\mathcal{U}(7,13)$ & $\mathcal{U}(30,50)$ & $\mathrm{nm} / \mathrm{s}$ \\
\hline \multirow{7}{*}{$\begin{array}{l}\text { Deformation } \\
\text { and motion }\end{array}$} & $F_{\text {tip }}$ & 25 & 12.5 & $\mu \mathrm{N}$ \\
\hline & $P_{c b}$ & 25 & 10 & $\mathrm{kPa}$ \\
\hline & $\gamma$ & 0.1 & 0.1 & $\mathrm{rad}$ \\
\hline & $E_{\varphi}$ & 1 & 1 & $\mathrm{kPa}$ \\
\hline & $E_{c}$ & 50 & 50 & $\mathrm{kPa}$ \\
\hline & $\nu_{\varphi}$ & 0.45 & 0.45 & \\
\hline & $\nu_{c}$ & 0.4 & 0.4 & \\
\hline \multirow[t]{11}{*}{ Texture } & $C_{c i}$ & $\mathcal{U}(1,2)$ & 1 & \\
\hline & $S_{c i}$ & 0.3 & 0.3 & \\
\hline & $F l_{c i}$ & 1 & 1 & \\
\hline & $F i_{c i}$ & 6 & 6 & \\
\hline & $C_{c b}$ & 2.5 & 2.5 & \\
\hline & $S_{c b}$ & 0.2 & 0.2 & \\
\hline & $F l_{c b}$ & 1 & 1 & \\
\hline & $F i_{c b}$ & 6 & 6 & \\
\hline & $Q$ & 64 & 64 & \\
\hline & $M_{\text {axial }}$ & 10 & 5 & \\
\hline & $\left|M_{\text {radial }}\right|$ & 12 & 4 & \\
\hline
\end{tabular}

The computer-generated image data, including its complete segmentation and tracking annotations, is made publicly available at http://cbia.fi.muni.cz/research/simulations/filogen.html.

\section{A. Simulation parameters}

The initial cell bodies were generated using the digital cell phantom simulator [23]. The parameter configurations used for (a)

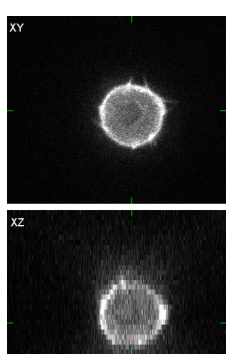

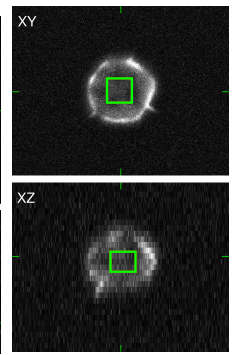

(b)

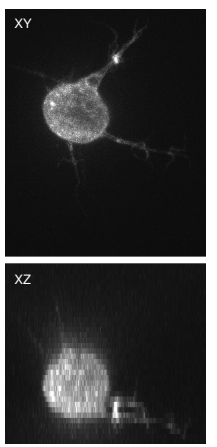

(c)



(d)
Fig. 6. A 3D visualization of real $(a, c)$ and synthetic cells (b, d) using XY and XZ slices. The overexpressing cells $(\mathrm{a}, \mathrm{b})$ have rather short filopodia, whereas the phospho-defective cells (c, d) have long filopodia with several branches. The overexpressing cells are visualized using single slices (green ticks show the selected slices). The phospho-defective cells are visualized using maximum intensity projections as the filopodia are long and difficult to see in a single slice. The green box in Fig. 6 b depicts a sample $31 \times 31 \times 3$ region of interest used for texture analysis.

generating synthetic 3D time-lapse sequences of both overexpressing and phospho-defective cells are listed in Table I. They reflect the diversity in the initial number $\left(N_{\varphi}\right)$, level of branching $\left(P_{b}\right)$, lifetime $\left(P_{a}\right.$ and $\left.P_{d}\right)$, shape, and dynamics of filopodia, as well as in the heterogeneity of cell body textures $\left(C_{c i}\right)$. In particular, the longer $\left(l_{0}\right)$ and thicker $\left(r_{m}\right.$ and $\left.r_{\varepsilon}\right)$ filopodia of the phospho-defective cells require less force to be bended $\left(F_{\text {tip }}\right)$ than those of the overexpressing cells. The number of actin filaments $\left(n_{f}\right)$ in the filopodia was chosen to be between 10 and 30, not to limit the filopodial growth by the plasma membrane resistance or insufficient free actin diffusion at filopodial tips [38]. The motion of both cell phenotypes was given by mimicking random-walk trajectories of the real cell centroids extracted from the available segmentations of the reference real image data [20]. In order to imitate a diversity of the cell texture among the individual real cells, the value of $C_{c i}$ parameter was generated for each overexpressing cell individually as a uniformly distributed random number.

Two synthetic 3D time-lapse sequences that were generated 
using the proposed simulation system with the listed parameter configurations are shown in Figs. 4 and 5, demonstrating the growth and motion of filopodia of a phospho-defective and an overexpressing cell, respectively. A qualitative comparison of the real and synthetic image data showing overexpressing and phoshodefective cells is given in Fig. 6. It can be seen that the textures of cell bodies and filopodia in the real and synthetic image data are visually comparable.

\section{B. Validation of synthetic image data}

The generated synthetic time-lapse datasets were thoroughly designed to aptly mimic the shape, size, internal structure, and filopodial tip dynamics of the reference real cells. To validate that our simulation system produces plausible image data, we performed several tests that quantitatively assess the similarity between the synthetic datasets and their real counterparts.

1) Texture of cell body: In order to avoid the influence of the background noise, we calculated gray level co-occurrence matrices over the cell body interior regions $(31 \times 31 \times 3$ voxels, see Fig. 6b) and followed the protocol introduced in [14]. In particular, five Haralick's texture descriptors: Texture contrast, Texture correlation, Texture homogeneity, Maximal correlation coefficient, and Entropy, were computed for all synthetic and real images, and their distributions, at the level of cell phenotypes, were mutually compared. To this end, the KolmogorovSmirnov test with quantile-quantile plots were used for a nonparametric comparison of the underlying distributions obtained for the real and synthetic image data (see Fig. 7).

The visual inspection of the quantile-quantile plots depicted in Fig. 7 reveals a high level of similarity because the points fit the straight line, representing the ideal identical distributions, most of the range. Except for one single case, all KolmogorovSmirnov tests retained the null hypothesis $(p>0.05)$, stating that the distribution of texture descriptors is the same in both groups. Note that the level of similarity reported here is higher than that reported in the previous study [14].

Despite an apparent texture heterogeneity in the real overexpressing cells, the results confirmed the ability of our system to imitate real cell body textures with comparable distributions of the evaluated texture descriptors. These descriptors computed for the synthetic phospho-defective cells were also comparable to the real ones, only their Entropy was slightly shifted to the lower values when compared to the distribution extracted from the real image data.

2) Motion of filopodial tips: To obtain the reference motion patterns of filopodial tips for each mimicked cell phenotype, three experts manually annotated spatio-temporal positions of individual filopodial tips available in the real image data. Next, the three created manual annotations were combined together by maximizing the overall Linear Oriented Forests Matching measure [39] between the combined, reference annotation and each of the three manual annotations. Finally, each reference trajectory (i.e., the trajectory of one particular filopodial tip in the reference annotation) was analyzed as described in [40]. In particular, we studied the orientations of the filopodial tip movement between two successive frames with respect to the primary trajectory direction. The possible orientations, always
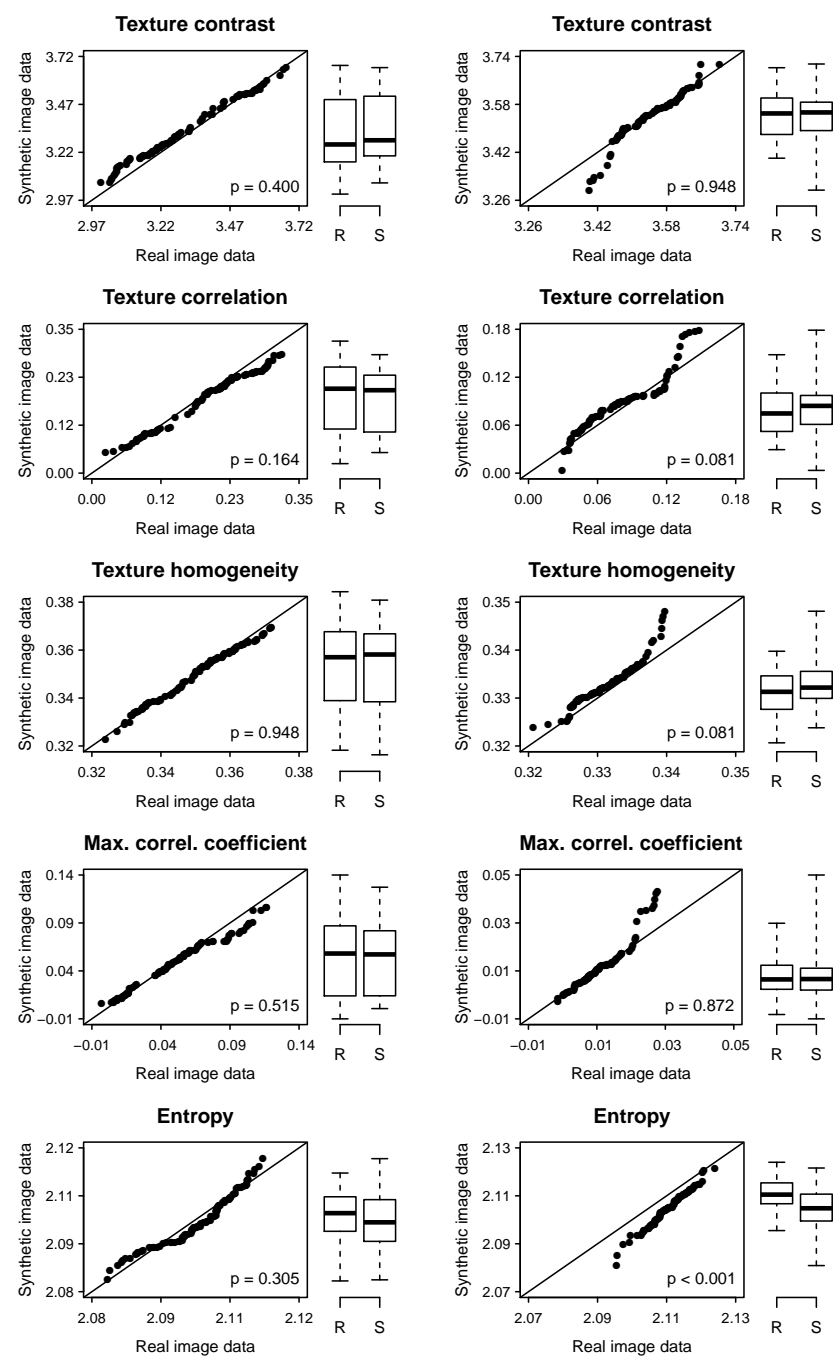

Fig. 7. Quantile-quantile plots and boxplots for the distributions of Haralick's textural descriptors computed over the cell body interior regions (see Fig. 6b) cropped from the real (R) and synthetic (S) image data of overexpressing (left column) and phospho-defective (right column) cells. The displayed quantiles are accompanied by the ideal identical distribution (straight line) and p-values of the Kolmogorov-Smirnov test. The whiskers of boxplots correspond to the minimum and maximum values, the boxes represent the interquartile ranges, and the thick marks stand for the median values.

falling in the $[0,180)$ interval, were binned into 12 subintervals with the width of 15 degrees (see Fig. 8). The reference motion patterns of filopodial tips for the overexpressing and phosphodefective cells were established by aggregating total distances within individual angular subintervals across all filopodial tips of either cell phenotype and by normalizing them to produce 1 when summed over all angular subintervals.

To assess the similarity of motion patterns of filopodial tips displayed in the synthetic image data, we applied the identical routine on its inherently generated reference annotations, and calculated the histogram intersection measure [41] between the motion patterns extracted from the real and synthetic image data of the particular cell phenotype (see Fig. 8). By inspecting the content of Fig. 8, it can be concluded that the proposed 

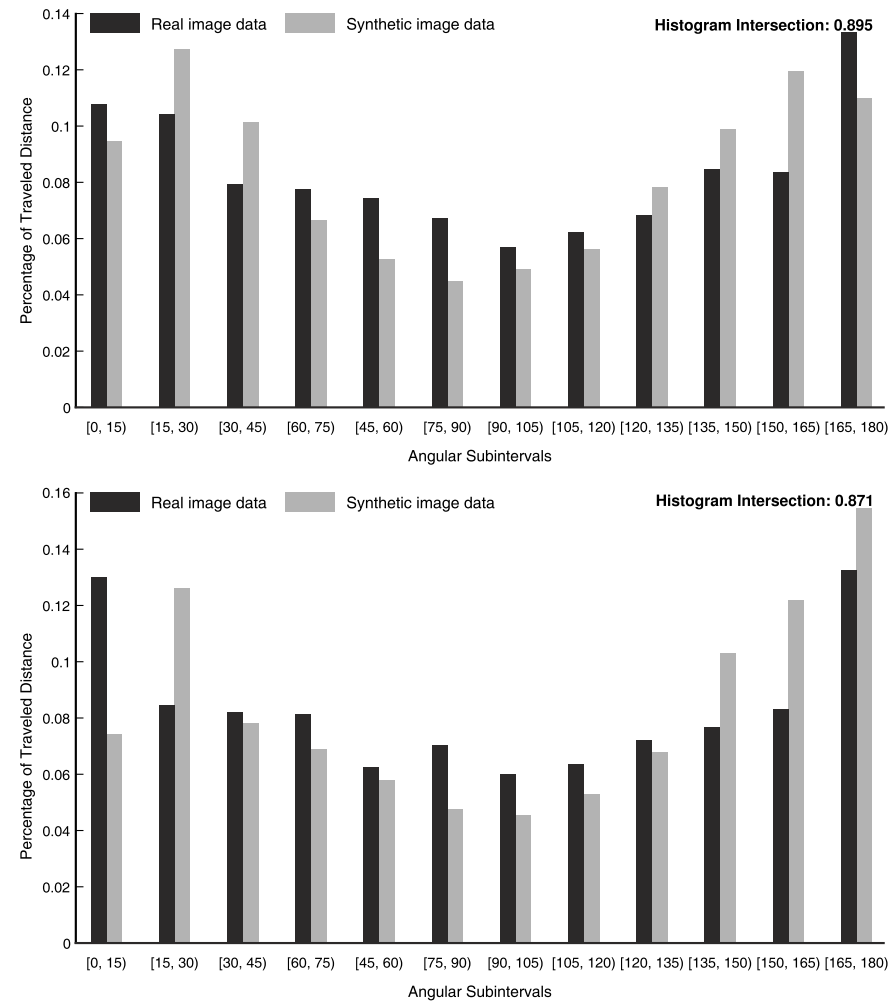

Fig. 8. The distance percentage traveled by all filopodial tips of overexpressing (upper) and phospho-defective cells (lower) in the real and synthetic image data at different orientations with respect to the primary trajectory direction of individual filopodial tips. The histograms are accompanied by the information on their similarity calculated using the histogram intersection measure.

simulation system is capable of plausibly mimicking random motion patterns of real filopodial tips, with the high degree of similarity exceeding the level of 0.87 on the $[0,1]$ scale.

\section{Implementation details and execution time}

The three modules and the supervisor involved in our simulation system were created as separate software components that exchange their data by reading from and writing to files. GM and the supervisor were implemented in Matlab R2017a. DM and TM were implemented in $\mathrm{C}++$, the former being built on the SOFA framework [42]. The modules and the supervisor were integrated into a pre-configured virtual machine, which is made publicly available, together with the source codes, at http://cbia.fi.muni.cz/research/simulations/filogen.html.

To provide the reader with an estimate of how long the generation of the synthetic image data took, we measured the execution time of each phase involved in the simulation process on a common workstation (Intel Xeon QuadCore $2.83 \mathrm{GHz}$, 32 GB RAM, Ubuntu 16.04 LTS).

The measured average execution times for generating one synthetic 3D image of a single overexpressing and phosphodefective cell are listed in Table II. By comparison with 346 seconds spent on average by one human expert when manually annotating one randomly selected slice for segmentation and the whole 3D image for filopodial tip tracking, it can be concluded that the proposed simulation system substantially reduces the time needed to establish a benchmarking dataset
TABLE II

THE AVERAGE EXECUTION TIME (IN SECONDS) OF EACH PHASE INVOLVED IN THE PROPOSED SIMULATION PROCESS FOR GENERATING ONE 3D IMAGE OF A SINGLE OVEREXPRESSING AND PHOSPHO-DEFECTIVE CELL.

\begin{tabular}{l|c|c|} 
Phase & Overpressing & Phospho-defective \\
\hline Geometry module & 20 & 173 \\
Deformation module & 8 & 3 \\
Texture module & 4 & 28 \\
Virtual microscopy & 12 & 36 \\
\hline Total & 44 & 240
\end{tabular}

of completely annotated image data for objective comparison of filopodium segmentation and tracking methods.

\section{CONCLUDING REMARKS}

We have presented a novel approach to generating synthetic 3D time-lapse sequences of single motile cells with filopodial protrusions of flexible structural and temporal attributes. Being composed of three globally synchronized modules, our system is capable of simulating the filopodium growth and morphology changes, modeling the joint motion and deformation of the cell body with a time-varying number of filopodia, and generating the realistic texture of the entire cell together with mimicking the process of fluorescence microscopy image formation. The filopodium evolution is modeled using stochastic simulations of filamentous growth on a molecular level. The joint motion and deformation of the cell body with multiple filopodia is built on the theory of elasticity and is efficiently implemented using the FEM. The realistic time-coherent cell texture is synthesized using the simulated fluorescent particles in the spatio-temporal domain, being periodically submitted to a virtual microscope.

The proposed simulation system was validated by comparing three generated 3D time-lapse sequences of single overexpressing and phospho-defective cells to their real counterparts. The highly flexible modules involved in our system allowed us to fine-tune the synthetic image data in a way to achieve high structural, visual, and quantitative similarity to the real image data in terms of cell body texture and filopodial tip motion.

Such a level of similarity to the real image data and the fact that our simulation system inherently generates completely annotated image data have stimulated the development of fully 3D bioimage analysis pipelines for filopodium segmentation and tracking [43], including those based on nowadays extremely popular machine-learning approaches that often suffer from a limited amount of annotated training data. Furthermore, the generation of such a synthetic dataset is significantly faster than the manual annotation of the real image data. Last but not least, our system is capable of generating synthetic image data of different spatio-temporal resolutions, which is something very difficult to achieve in real microscopy setups due to their physics-based limitations. Such a feature may help in objective benchmarking of the developed bioimage analysis algorithms and in deeper understanding of their limitations, thus providing users as well as algorithm developers with practical clues on the algorithm behavior.

In spite of being flexible and mathematically sound, our system has its limitations that stem from the design of individual 
modules. This is particularly true for GM and DM. The former has been designed to simulate the evolution of filopodia, barely allowing straightforward modifications that would facilitate to model the evolution of other types of cell protrusions, such as blebs. Furthermore, the current model of filopodial branching, being built on a random process, could possibly be improved by modeling the dynamics of cross-linking proteins to control the bundling and deviation of actin filaments in a biologically more relevant fashion [44]. The latter module strictly requires the modeled cell geometries to be decomposable into a reasonable number of volumetric elements that are supported by the SOFA framework [42], and to behave according to the theory of elasticity that forms widely acceptable principles of plasma membrane dynamics of diverse cell phenotypes [45]. Thus, the requirement for such elastic behavior may allow one to mimic sophisticated cell migration modes, such as mesenchymal and amoeboid motility [46]. Finally, concerning TM, the clouds of simulated fluorescent particles can represent a broad range of fluorescent reporters [14]. Furthermore, their spatial resolution is not bound to any particular image grid, which allows one to interconnect this texture model with spatially high-resolution models of cell shape development [47]-[49].

In our future work, we intend to further extend and adapt the powerful mathematical models employed in all three modules of our simulation system, with the primary aim of simulating scenarios with multiple, mutually interacting cells with filopodial protrusions, which exhibit different cell migration modes and control filopodial branching via insufficient concentrations of cross-linking proteins within the bundles of actin filaments.

\section{ACKNOWLEDGMENT}

The authors would like to thank X. Morales and A. Rouzaut (Center for Applied Medical Research, Spain) for providing the real image data of overexpressing and phospho-defective cells and for their valuable comments on the biological background of the CRMP-2 protein and its mutations.

\section{REFERENCES}

[1] P. K. Mattila and P. Lappalainen, "Filopodia: Molecular architecture and cellular functions," Nature Reviews Molecular Cell Biology, vol. 9, no. 6, pp. 446-454, 2008.

[2] M.-F. Carlier, Actin-based Motility. Heidelberg, Germany: SpringerVerlag, 2010.

[3] T. Shibue, M. W. Brooks, M. F. Inan, F. Reinhardt, and R. A. Weinberg, "The outgrowth of micrometastases is enabled by the formation of filopodium-like protrusions," Cancer Discovery, vol. 2, no. 8, pp. 706$721,2012$.

[4] A. Haeger, K. Wolf, M. M. Zegers, and P. Friedl, "Collective cell migration: Guidance principles and hierarchies," Trends in Cell Biology, vol. 25, no. 9, pp. 556-566, 2015.

[5] G. Jacquemet, H. Hamidi, and J. Ivaska, "Filopodia in cell adhesion, 3D migration and cancer cell invasion," Current Opinion in Cell Biology, vol. 31 , no. 10 , pp. 23-31, 2015.

[6] C. Ortiz-de-Solórzano, A. Muñoz-Barrutia, E. Meijering, and M. Kozubek, "Toward a morphodynamic model of the cell," Signal Processing Magazine, vol. 32, no. 1, pp. 20-29, 2015.

[7] A. Cardona and P. Tomancak, "Current challenges in open-source bioimage informatics," Nature Methods, vol. 9, no. 7, pp. 661-665, 2012.

[8] A. E. Carpenter, L. Kamentsky, and K. W. Eliceiri, "A call for bioimaging software usability," Nature Methods, vol. 9, no. 7, pp. 666-670, 2012.

[9] M. Kozubek, "Challenges and benchmarks in bioimage analysis," in Advances in Anatomy, Embryology and Cell Biology. Springer, Cham, 2016, vol. 219, pp. 231-262.
[10] D. L. Coutu and T. Schroeder, "Probing cellular processes by long-term live imaging-historic problems and current solutions," Journal of Cell Science, vol. 126, no. 17, pp. 3805-3815, 2013.

[11] N. Chenouard, I. Smal, F. de Chaumont, M. Maška et al., "Objective comparison of particle tracking methods," Nature Methods, vol. 11, no. 3, pp. 281-289, 2014.

[12] M. Maška, V. Ulman, D. Svoboda et al., "A benchmark for comparison of cell tracking algorithms," Bioinformatics, vol. 30, no. 11, pp. 16091617, 2014.

[13] V. Ulman, M. Maška, K. E. G. Magnusson et al., "An objective comparison of cell-tracking algorithms," Nature Methods, vol. 14, no. 12, pp. 1141-1152, 2017.

[14] D. Svoboda and V. Ulman, "Mitogen: A framework for generating 3D synthetic time-lapse sequences of cell populations in fluorescence microscopy," IEEE Transactions on Medical Imaging, vol. 36, no. 1, pp. 310-321, 2017.

[15] D. Tsygankov, C. G. Bilancia, E. A. Vitriol, K. M. Hahn, M. Peifer, and T. C. Elston, "CellGeo: A computational platform for the analysis of shape changes in cells with complex geometries," Journal of Cell Biology, vol. 204, no. 3, pp. 443-460, 2014.

[16] D. J. Barry, C. H. Durkin, J. V. Abella, and M. Way, "Open source software for quantification of cell migration, protrusions, and fluorescence intensities," Journal of Cell Biology, vol. 209, no. 1, pp. 163-180, 2015.

[17] V. Urbančič, R. Butler, B. Richier, M. Peter, J. Mason, F. J. Livesey, C. E. Holt, and J. L. Gallop, "Filopodyan: An open-source pipeline for the analysis of filopodia," Journal of Cell Biology, vol. 216, no. 10, pp. 3405-3422, 2017.

[18] G. Jacquemet, I. Paatero, A. F. Carisey, A. Padzik, J. S. Orange, H. Hamidi, and J. Ivaska, "FiloQuant reveals increased filopodia density during breast cancer progression," Journal of Cell Biology, vol. 216, no. 10, pp. 3387-3403, 2017.

[19] D. V. Sorokin, I. Peterlík, V. Ulman, D. Svoboda, and M. Maška, "Model-based generation of synthetic 3D time-lapse sequences of motile cells with growing filopodia," in IEEE Internationl Symposium on Biomedical Imaging, 2017, pp. 822-826.

[20] M. Maška, X. Morales, A. Muñoz-Barrutia, A. Rouzaut, and C. Ortiz-deSolórzano, "Automatic quantification of filopodia-based cell migration," in IEEE International Symposium on Biomedical Imaging, 2013, pp. $668-671$.

[21] Y. Lan and G. A. Papoian, "The stochastic dynamics of filopodial growth," Biophysical Journal, vol. 94, no. 10, pp. 3839-3852, 2008.

[22] C. A. Felippa, "A systematic approach to the element independent corotational dynamics of finite elements," Cent. for Aerosp. Str., Tech. Rep. CU-CAS-00-03, 2000.

[23] D. Svoboda, V. Ulman, and I. Peterlík, "On proper simulation of chromatin structure in static images as well as in time-lapse sequences in fluorescence microscopy," in IEEE International Symposium Biomedical Imaging, 2015, pp. 712-716.

[24] V. Ulman, D. Svoboda, M. Nykter, M. Kozubek, and P. Ruusuvuori, "Virtual cell imaging: A review on simulation methods employed in image cytometry," Cytometry Part A, vol. 89, no. 12, pp. 1057-1072, 2016.

[25] R. Erban and J. S. Chapman, "Reactive boundary conditions for stochastic simulations of reaction-diffusion processes," Physical Biology, vol. 4, no. 1, p. 16, 2007.

[26] R. Erban, J. S. Chapman, and P. Maini, "A practical guide to stochastic simulations of reaction-diffusion processes," arXiv preprint arXiv:0704.1908, 2007.

[27] J. S. Przemieniecki, "Matrix structural analysis of substructures," AIAA J., vol. 1, no. 1, pp. 138-147, 1963.

[28] S. Cotin, C. Duriez, J. Lenoir, P. Neumann, and S. Dawson, "New approaches to catheter navigation for interventional radiology simulation," in Int. Conf. MICCAI, 2005, pp. 534-542.

[29] D. Baraff and A. Witkin, "Large steps in cloth simulation," in Proceedings of the 25th annual conference on Computer graphics and interactive techniques. ACM, 1998, pp. 43-54.

[30] I. Peterlik, M. Nouicer, C. Duriez, S. Cotin, and A. Kheddar, "Constraint-based haptic rendering of multirate compliant mechanisms," IEEE Transactions on Haptics, vol. 4, no. 3, pp. 175-187, 2011.

[31] M. Nesme, Y. Payan, and F. Faure, "Efficient, physically plausible finite elements," in Eurographics, 2005, pp. 77-80.

[32] I. Peterlík, C. Duriez, and S. Cotin, "Modeling and real-time simulation of a vascularized liver tissue," in Int. Conf. MICCAI, 2012, pp. 50-57.

[33] J. Ghaye, G. Micheli, and S. Carrara, "Simulated biological cells for receptor counting in fluorescence imaging," BioNanoScience, vol. 2, pp. 94-103, 2012. 
[34] K. Perlin, "An image synthesizer," in SIGGRAPH '85: Proceedings of the 12th annual conference on Computer graphics and interactive techniques. New York, USA: ACM Press, 1985, pp. 287-296.

[35] C. Rousselle, S. Paillasson, M. Robert-Nicoud, and X. Ronot, "Chromatin texture analysis in living cells," The Histochemcal J., vol. 31, no. 1 , pp. 63 - 70, 1999.

[36] D. Svoboda, M. Kozubek, and S. Stejskal, "Generation of digital phantoms of cell nuclei and simulation of image formation in $3 \mathrm{D}$ image cytometry," Cytometry Part A, vol. 75A, no. 6, pp. 494-509, 2009.

[37] D. Svoboda, V. Ulman, L. Matyska, M. Maška, J. Bella, and S. Stejskal, "On proper simulation of phenomena influencing image formation in fluorescence microscopy," in 2014 IEEE International Conference on Image Processing (ICIP), Oct 2014, pp. 3944-3948.

[38] A. Mogilner and B. Rubinstein, "The physics of filopodial protrusion," Biophysical Journal, vol. 89, no. 2, pp. 782-795, 2005.

[39] M. Maška and P. Matula, "Particle tracking accuracy measurement based on comparison of linear oriented forests," in IEEE International Conference on Computer Vision Workshops, 2017, pp. 11-17.

[40] P.-H. Wu, A.Giri, and D. Wirtz, "Statistical analysis of cell migration in $3 \mathrm{D}$ using the anisotropic persistent random walk model," Nature Protocols, vol. 10, no. 3, pp. 517-527, 2015.

[41] S.-H. Cha and S. N. Srihari, "On measuring the distance between histograms," Pattern Recognition, vol. 35, no. 6, pp. 1355-1370, 2002.

[42] F. Faure, C. Duriez, H. Delingette, J. Allard, B. Gilles, S. Marchesseau, H. Talbot, H. Courtecuisse, G. Bousquet, I. Peterlik et al., "Sofa: A multi-model framework for interactive physical simulation," in Soft Tissue Biomechanical Modeling for Computer Assisted Surgery. Springer, 2012, pp. 283-321.

[43] C. Castilla, M. Maška, E. Meijering, D. V. Sorokin, and C. Ortiz-deSolorzano, "Three-dimensional lifetime quantification of filopodia in migrating cancer cells," Submitted to IEEE Transactions on Medical Imaging, April 2018.

[44] Y. S. Aratyn, T. E. Schaus, E. W. Taylor, and G. G. Borisy, "Intrinsic dynamic behavior of fascin in filopodia," Molecular Biology of the Cell, vol. 18, no. 10, pp. 3928-3940, 2007.

[45] G. Danuser, J. Allard, and A. Mogilner, "Mathematical modeling of eukaryotic cell migration: Insights beyond experiments," Annual Review of Cell and Developmental Biology, vol. 29, no. 1, pp. 501-528, 2013.

[46] M. K. Driscoll and G. Danuser, "Quantifying modes of 3D cell migration," Trends in Cell Biology, vol. 25, no. 12, pp. 749-759, 2015.

[47] D. Shao, H. Levine, and W.-J. Rappel, "Coupling actin flow, adhesion, and morphology in a computational cell motility model," Proceedings of the National Academy of Sciences, vol. 109, no. 18, pp. 6851-6856, 2012.

[48] C. J. Cyron, K. W. Müller, A. R. Bausch, and W. A. Wall, "Micromechanical simulations of biopolymer networks with finite elements," Journal of Computational Physics, vol. 244, pp. 236-251, 2013.

[49] J. Prost, F. Jülicher, and J. Joanny, "Active gel physics," Nature Physics, vol. 11 , no. 2 , pp. $111-117,2015$. 